\title{
Exploring the Effects of Contextual Factors on Home Lighting Experience
} Jooyoung Jung ${ }^{1}$, Kwangmin $\mathrm{Cho}^{2}$, Soyoung $\mathrm{Kim}^{2}$, Chajoong $\mathrm{Kim}^{2 *}$

${ }^{1}$ Department of Industrial Design, Delft University of Technology, Delft, the Netherlands ${ }^{2}$ School of Design \& Human Engineering, UNIST, Korea

\begin{abstract}
Background Although lighting increasingly penetrates our everyday life due to technology advancement, little is known about how people interact with lighting and how contextual factors impact on the experience. Thus, this study attempted to reveal how two contextual factors (the level of concentration required for pleasant lighting use and social interaction) could influence the manipulation of lighting parameters, particularly focusing on the major factors of lighting such as illuminance, color temperature, and hue.
\end{abstract}

Methods To understand of the interaction between contextual factors and lighting variables, an experiment was conducted. 10 singles and 10 couples had to manipulate lighting variables such as intensity and colorin five everyday situations for pleasant lighting experience.

Results The result of the experiment showed that illuminance, color temperature and hue are influenced by the degree of concentration, but only partially influenced by social factors. The findings could provide a better understanding of manipulating lighting variables in terms of use context with design practitioners.

Conclusions The overall findings of the study indicate that illuminance, color temperature, and hue are significantly dependent upon the level of concentration required in at-home lighting use, and also have only a partial dependence on social effect. This implies that although we assumed that people have their personal lighting preferences, their preferences can be largely dependent on the degree of concentration required for at-home pleasant lighting use. Hence, there are common patterns among people in manipulating lighting parameters, which are less dependent on personal differences.

Keywords Lighting, User Experience, Situation, Concentration, Social Effect

* Corresponding author: Chajoong Kim (cjkim@unist.ac.kr)

This work was supported by the National Research Foundation of Korea Grant funded by the Korean Government (NRF2014S1A5A8019577).

Citation: Jung, J., Cho, K., Kim, S., \& Kim, C. (2018). Exploring the Effects of Contextual Factors on Home Lighting Experience. Archives of Design Research, 31(1), 5-21.

http://dx.doi.org/10.15187/adr.2018.02.31.1.5

Received : Jan. 16. 2018 ; Reviewed : Jan. 26. 2018 ; Accepted : Jan. 26. 2018

pISSN 1226-8046 eISSN 2288-2987

Copyright : This is an Open Access article distributed under the terms of the Creative Commons Attribution NonCommercial License (http://creativecommons.org/licenses/by-nc/3.o/), which permits unrestricted educational and non-commercial use, provided the original work is properly cited. 


\section{Introduction}

Among the human senses, vision plays a dominant role in human perception. More than $50 \%$ of our brain is directly or indirectly related to vision (Elizabeth, 1996). Visual stimulation begins when light from an object is reflected into the eyes. The signal is transferred to the brain and people recognize the object. The sun is usually our main source of illumination throughout the day (Sanders, 1993). However, in case of lack of light in space (i.e. in darkness or at night), visual perception is getting more difficult. In this situation, artificial light plays an important role in increasing perceptual chances. The notion of lighting means the use of light, which includes both natural illumination by capturing daylight and artificial light sources such as lamps and light fixtures. Since light bulbs were invented, our daily activities have been extended to late night, and we have been able to work in dark places such as mines or even the deep sea easier than ever before. Lighting has primarily functioned to brighten spaces, which is defined as visibility (Goldstein, 2009). However, as new lighting sources were developed and became more controllable, the role of lighting has been diversified. For instance, in recent years, much attention has been paid to LED (Lighting Emitting Diode) because it is more energy efficient, digitally controllable and much smaller than the earlier types of a lamp (Hoonhout, Jumpertz, \& Mason, 2011). With the advent of LED, lighting does not only function to illuminate, but also to transfer information, enhance visual aesthetics, and induce emotions(Figure 1)(Jun et al., 2014).

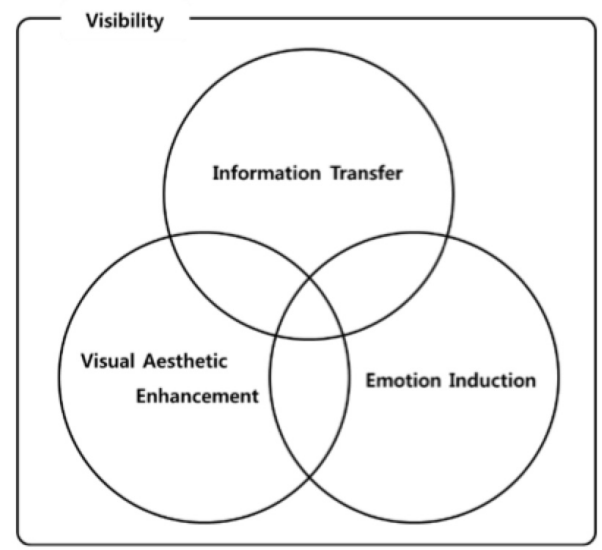

Figure 1 The visibility and three sub-functions of lighting in the human-lighting interaction

Besides visibility (the essential function of lighting), lighting can provide people with information through visual stimuli. A typical example is traffic signals. Drivers and pedestrians recognize when to go and stop through colors given by the traffic lights, which work to transfer information. Lighting can also provide information by helping users to recognize the status of products. For instance, the LED indicator, embedded in a digital camera battery charger, shows through color or blinking how much the battery is charged. An object can look aesthetically nicer with the help of lighting. For instance, since the fifteenth-century chandeliers have been a symbol of luxury with decorative profusion and an exhibition of wealth (McCaffety, 2006). Many desk lamps function as decorative elements in 
the home, next to the primary role of brightening the space (Choi et al., 2007). Furthermore, lighting affects the emotion of people. By controlling the characteristics of lighting based on understanding the process of human visual perception, lighting can affect the emotion that people experience in space (Essig \& Setlow, 2012). For instance, in the theatre the goal of lighting is completely focused on creating an immersive illusion and atmosphere that enhance the storytelling experience of the spectator through the interplay of light and shadow, colour and texture (Blühm \& Lippincott, 2001). Although the responses to color lighting could differ between people, certain colors tend to lead to similar emotions. For example, lighting in red is often associated with passion or anger, particularly in theatre settings (Essig\& Setlow, 2012). In our everyday life, however, lighting is experienced as the integration of the roles rather than one role only. For instance, light from a bedside lamp makes the bedroom look relaxing (emotion induction) and more beautiful (visual aesthetic enhancement) while showing it is time to sleep to people outside the room (information transfer). The identification of the roles of lighting led us to the question: what kinds of parameters of lighting have been identified, which can be used to enhance visual aesthetic of the space, evoke particular emotions and transfer information.

The parameters of lighting that affect vision have been diversified. Lighting has played a key role in theatre to create touching scenes in drama. Initially, four parameters of lighting were considered in theater lighting: distribution, intensity, movement, and color (Gillette, 2012). Recently, Essig and Setlow (2012) have identified seven parameters of lighting: intensity, color, direction, distribution, texture, shape, and movement. As lighting technologies are advanced, especially interactive design study related to lighting also has described seven parameters: the number of lighting, intensity, amount of light, type of light, color, reaction scheme, and light changes (Chang, 2010). Whereas architecture and interior design focus more on the arrangement or position of lighting than the other basic parameters of lighting. These studies of theatre and interaction have shown that many parameters of lighting are adjustable and these parameters would transfer diverse visual perception to human. Bertin's research has represented the original visual variables and has described seven visual variables that these units were modified: value (change from light to dark), color size (change in length, area and repetition), texture (variation in grain), shape (infinite number of shapes), position (change in the location) and orientation (changes in alignment)(Bertin, 1983). Because the meanings of lighting parameters look similar or overlapping among studies, redefined lighting parameters were necessary for the study, through which existing lighting parameters can be overall and clearly represented. Based on the literature review, seven parameters of lighting could be redefined: intensity (the level of illuminance), color (consisting of color temperature and hue as stimulation in the retina by spectrum of light), area (illumination area by lighting), movement (automated changes of lighting), texture (the degree to which light scatters) and distribution (the direction of emitted light)(Figure 2) (Jun et al., 2014): 
Intensity: it means the amount of light energy or a level of brightness as the physical property of lighting. The unit of intensity ranges from weakness to strength.

Color: it is the results of stimulation in the retina by the spectrum of light. White light such as the sun includes all visible continuous spectrum from approximately 700 to 400 nanometers. On the other hand, monochromatic light includes certain spectrum, and it looks like only one color light.
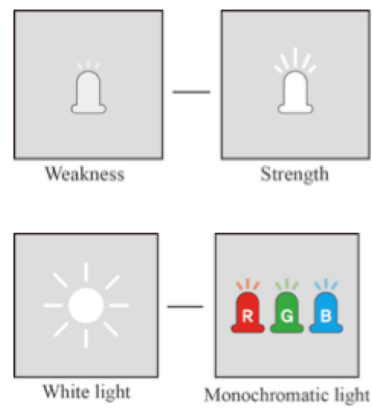

Area: it means the illumination area, and it is divided into narrow or wide. The emitting area changes according to the number of lightings.

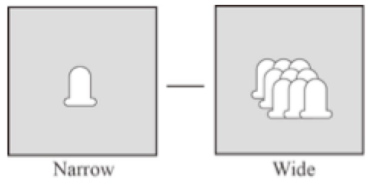

Movement: it means automated changes of lighting in color, intensity and so on. Blinking lighting is one of the quintessences in movement. One range of movement is static that is equal and monotonic lighting. The other range is dynamic lighting.

Texture: it is the degree of light scattering, and it is described as hardness or softness. Hardness means dazzling light because of its convergence, and softness means blurred and delicate light.

Distribution: it means the method according to a direction of the light emitting. The direct one is used to spot objects directly and the light source could be seen in a spectator's position. On the other hand, the indirect one does not expose the light source straightly to a spectator and it creates diffuse and reflected light.
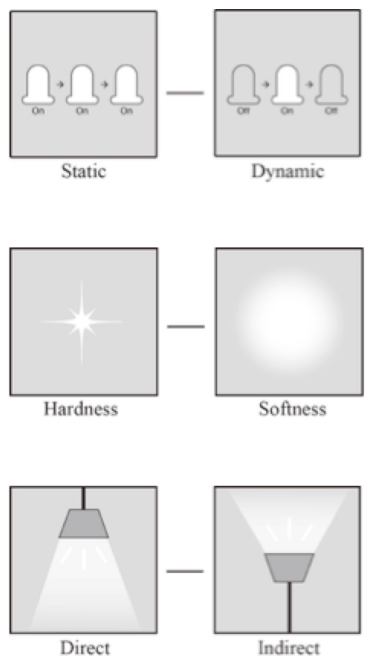

Shape: it refers to an arrangement, a form of surrounding ornament, and the outer of light source change shape. Lighting shape is divided into simple and complexity state.

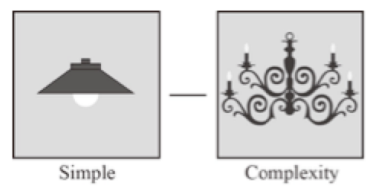

Figure 2 Seven parameters of lighting in the study

According to Offermans et al. (2014), people have different levels of lighting needs that are highly dependent on context. This differences in the level result from a high degree of control over various lighting parameters such as illuminance, colour, focus, and hue. As a result, this allows people to create more suitable atmospheres for the situation they are in and as such have different lighting settings for different contexts. Especially, as our everyday environments are becoming more and more dynamic, they are used for a wider variety of activities: e.g., spaces traditionally for the domain of family life often become workspaces and vice versa. Emphasizing the dynamic situations on a daily basis, Magielse (2011) argued that the social influence of lighting needs to be taken into consideration to make adaptive lighting environment viable. Therefore, our assumption in the study is that the usage of lighting is influenced by the context in which the lighting is used. The context of lighting use consists of 
two elements in a space, the level of concentration required in lighting use and whom the user is with. If this is the case, in what way do the two elements influence the use of lighting? The user sets up the parameters of lighting depending on the degree of concentration required to do an activity and whom he/she is with: defined as the level of concentration and social effect respectively. However, only a few studies have been conducted about how people control lighting given the level of concentration and social interaction. Therefore, based on the understanding of the role and parameters of lighting, we attempted to reveal how the level of concentration and social context influence the manipulation of lighting parameters. Only intensity (i.e., illuminance) and color (i.e., color temperature and hue) among seven lighting parameters were taken into consideration because they are the parameters of lighting that are most common and are used mainly in the home context. Figure 3 shows the conceptual framework of the study.

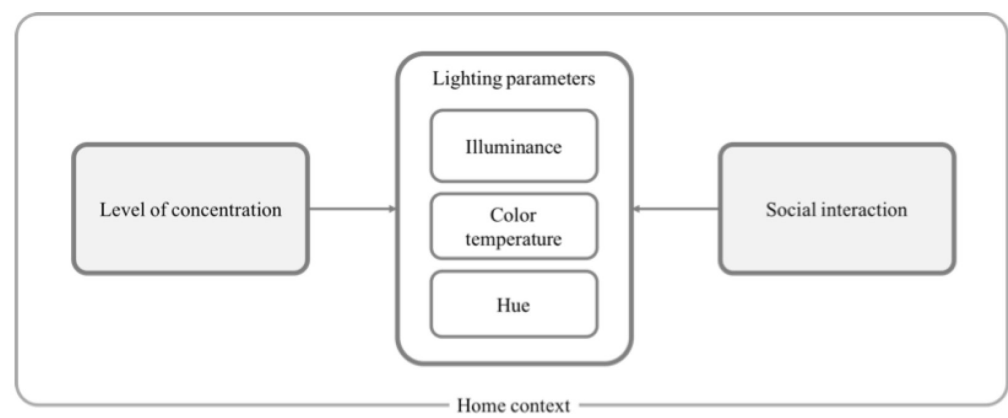

Figure 3 The conceptual framework of the study

\section{Methods}

\section{1. At-home lighting use according to the level of concentration}

The study focused on how lighting parameters are influenced by particular context where lighting is used. The home was taken into account as the target context for this study because in our everyday life lighting is often used at home in the evening or at night while various activities are taking place at the place,such as cooking, taking a bath, reading a book, watching TV, going to bed, and so on. In order to determine situations in which people use lighting at home, a brainstorming session with the researchers as well as three masters students whose background is industrial design was conducted to figure out possible situations in association with lighting use at home. In the session, we collected multiple activities that people do at home on the internet and then discussed which activities were done by most of the people with regard to at-home lighting experience. It turned out that lighting-involved activities at home could be characterized in terms of 'pleasant-unpleasant' and 'focused and unfocused'. For instance, reading a cartoon under a lamp is pleasant while doing an assignment negative. Likewise, watching TV is focused while listening to music is less focused. In the study, the focus was on pleasant activities because in the home context lighting is associated with facilitating more positive mood than negative mood and social interaction is closely linked to pleasant lighting experience. Therefore, the activities were 
determined regarding the extent to which concentration is required to do the activity among positive lighting experiences which are done by the majority of people (Figure 4).

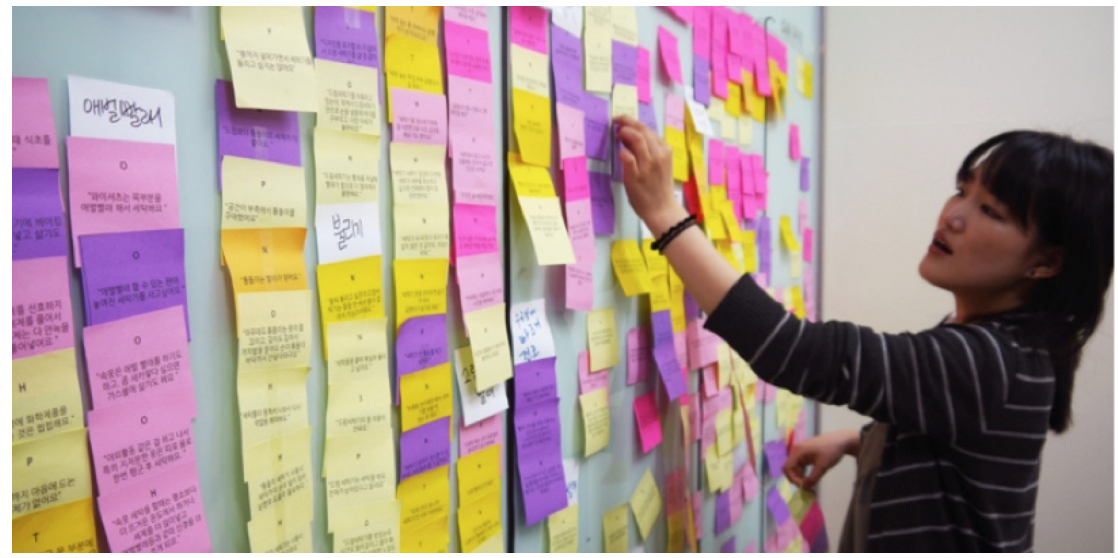

Figure 4 the brainstorming session to selectat-home lighting use situations

This led to the identification of two categories of situations in terms of concentration in which lighting plays a major role: activities requiring a high level of concentration (playing Sudoku and reading a magazine) and activities requiring a low level of concentration (having dinner and taking a break). In the situations, watching YouTube has additionally included apart from playing Sudoku and reading a magazine because the activity at home is getting more and more popular with smartphone or tablet PC generalisation (Figure 5).

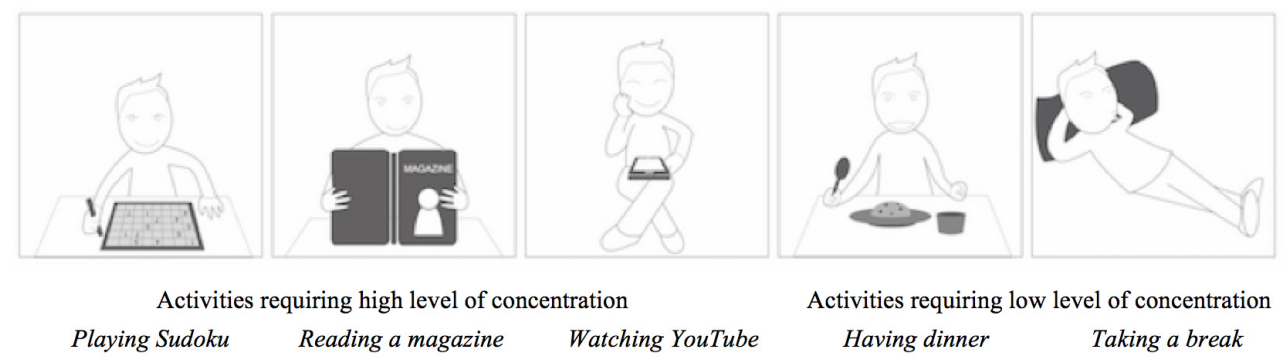

Figure 5 Selected five at-home acitivities in relation to lighting use in terms of level of concentration

\section{2. Sample}

The experiment comprised of two sessions: one aimed at revealing how lighting parameters are influenced by the level of concentration required in situations, and the other focused on the social effects of lighting usage. It was to see how the usage of lighting parameters are influenced by a couple of users staying in a space compared with the usage by a single user. In the first session, 10 single participants were recruited, consisting of 5 males and 5 females to eliminate a possible bias by gender (i.e., the gender effect). In the second session, 10 couples were recruited as participants, who did not participate in the first session. The couple means man and woman having had a relation in real life at the time of the experiment. The age of both groups ranged between 20 and 29 years old, and they were all university students. After the experiment, they were paid for the participation. All of them were those who had no vision problems such as color-blindness or color amblyopia. All the participants in both 
sessions were recruited through advertisements on social media such as Facebook in which general information such as the aim of the study and the target sample was described.

\section{3. Instruments}

The experiment was conducted in Home Lab at UNIST, where a living room and a dining room are designed to make participants feel comfortable as if they were at home (Figure 6). Four IP cameras, connected to the computer in the control room of Home Lab, were installed on the ceiling of Home Lab to observe and, at the same time, to videotape the experiment. Beforehand, consent of all participants was received regarding videotaping during the experimental sessions.

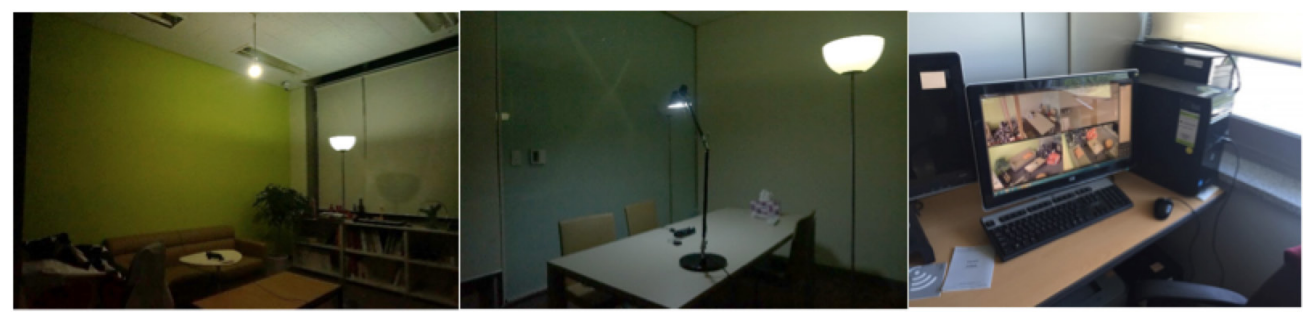

Figure 6 Home Lab at UNIST:

the living room (left), the dining room (middle) and the control room (right)

Four Philips Hue light bulbs were installed in Home lab: two inlighting fixtures in the living room; one of which is indirect as well as portable lighting, and two in the lighting fixtures in the dining room (Figure 7).
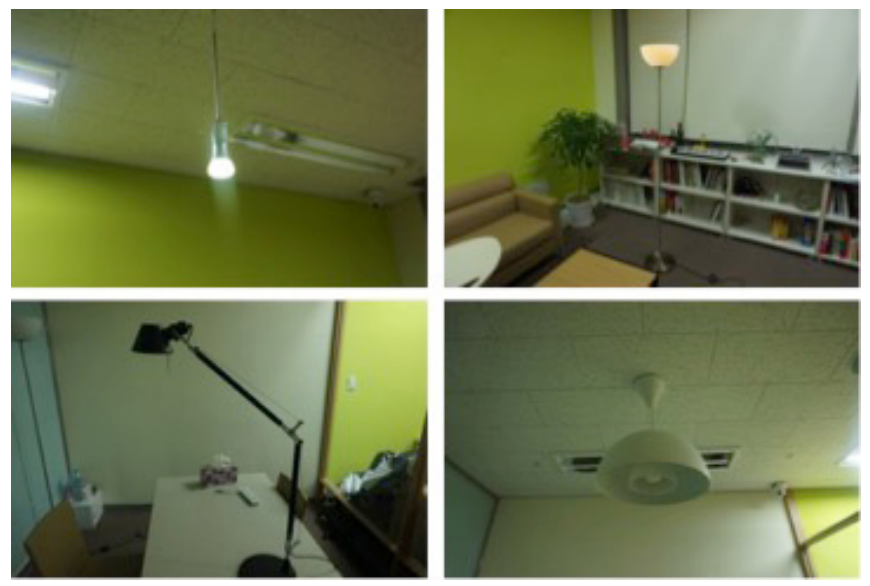

Figure 7 Philips Hue LED light bulbs installed in Home Lab connected to the bridge: ceiling lighting (upper left), portableindirect lighting (upper right) at the living room, and desktop lighting (lower left) and dining lighting (lower right) at the dining room

The illuminance, color temperature, and hue (color) of lightings could be controlled in real time through a smartphone using the Philips Hue application. An illuminometer was also used to get the quantitative data of illuminance and color temperature of where the user was located (Figure 8). 

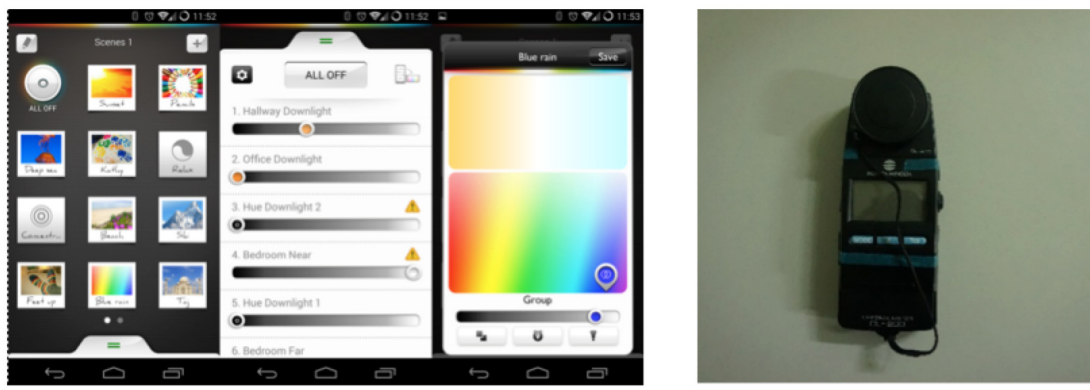

Figure 8 The Philips Hue application (left)and the illuminometer (right) used in the experiment

In order to figure out the reasons of controlling lighting parameters for tasks, an interview question was formulated, what made you set up that particular lighting condition? For each of the five at-home activities, required materials were prepared: a piece of paper on which sudoku is printed with pencil and eraser, lifestyle magazines, sandwiches with a cup of juice and Fatboys (see Table 1). An iPad was also required to play YouTube. It would influence on the situation of the lighting because it gives its own light. In order to minimize the influence of emotion induced by YouTube video itself, a TED video on science was selected for the experiment.

Table 1 Materials and tasks for each situation

\begin{tabular}{llllll} 
& Task 1 & Task 2 & Task 3 & Task 4 & Task 5 \\
Materials & Sudoku, pen & Magazines & $\begin{array}{l}\text { The link of a TED } \\
\text { video on science }\end{array}$ & $\begin{array}{l}\text { Sandwiches, dish, Fatboy } \\
\text { juice }\end{array}$ \\
\cline { 2 - 6 } & & & &
\end{tabular}

\section{4. Procedure}

First, participants were invited to Home lab. Before the start of the experiment, the session moderator introduced the aim of the experiment to each participant. Next, the participant/ couple were asked to install the Philips Hue application on their own smartphone. They were given time to practice the app. Additional instruction was available if needed. Participants were then left alone in Home Lab with a sheet of tasks.

Following the procedure is written in the instruction sheet, the participant was shown the first task. They were first asked to configure the illuminance, color temperature and hue of lighting with the Philips Hue app, which best suited him/her for that task (see an example of a couple participants in Figure 9). They were allowed to adjust the light variables themselves during the task. Then, the participant(s) accomplished that task. The same procedure was repeated for the other four tasks. The order of the five tasks was randomized in order to avoid any sequence effect. No time limit was given to finish each task aiming to give sufficient time to prompt perceptions of the environment and find the best lighting setting for each task. In case a task could not be completed (for example, when they couldn't set up lighting variables 
with Philips Hue), we intervened and guided them to finish the task successfully. After the session, a retrospective interview was conducted which focused on identifying the reasons for having chosen the particular lighting in each task. The session with single participants was conducted first, which was followed by the experiment with participant couples on another day. In order to maximize the social effect in the experiment, couples were encouraged to talk in a natural way to each other during task performance. All sessions were observed and videotaped from the control room. To get quantitative data of the light configuration installed by the participants for each task, a screenshot of the application was taken whenever they finished the set-up of the lighting: a screenshot contained the information of the light settings for each task. After they left the room, the lighting variables for each task were reproduced by setting up Philips Hue according to the screenshot. The color values of the light settings were then measured from the position of the participant(s) in the room with the illuminometer.
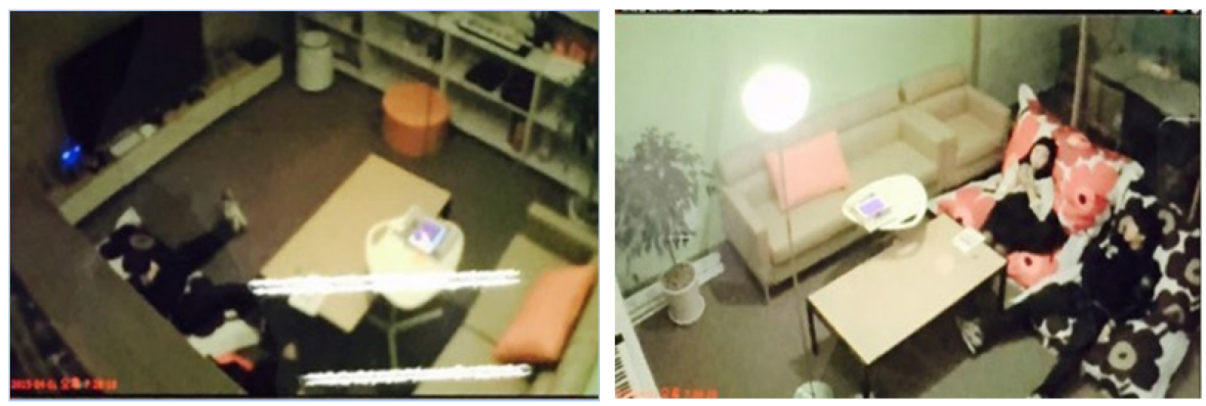

Figure 9 A scene of a couple's taking a rest in the experiment

\section{5. Data analysis}

A Friedman test was conducted in order to figure out how illuminance, color temperature, and hue are influenced by given tasks. The task is the independent variable and the values of illuminance, color temperature, and hue are the dependent variables. For this, the data from both 10 single participants and 10 couple participants was used. A t-test analysis was also carried out between the single participant group and the couple participant group to see how social effect plays a role in lighting usage. Because the hue data from the experiment can be illustrated only in color coordinate, they were graphically analysed instead of statistical analysis. The data from both 10 single participants and 10 couple participants were compared for the statistical analysis. The qualitative data from the retrospective interviews were then categorized in terms of similarity and quantified in order to explain why the participants had configured particular lightings parameters between tasks and also to explain how social factor affects on lighting usage.

\section{Results}

\section{1. Illuminance and color temperature between tasks}

Illuminance, also called brightness perceived by human, refers to how much the light illuminates the space. A high value of illuminance means space is bright while a low value of illuminance is meant to be dim. Participants could have freedom to select a value between 
o and 200 lux with Philip Hue. The mean values of their selection were from 4.47 lux to 133.92 depending on the task. According to the results of the statistical analysis, there are statistically significant differences in both illuminance and colour temperature: illuminance with a Chi-square value of 68.32 ( $\mathrm{p}<.01)$, and colour temperature with a Chi-square value of $24.61(\mathrm{p}<.01)$. This indicates that the mean values of illuminance and color temperature are dependent on the task. For illuminance, playing Sudoku has the highest mean value, while watching YouTube and taking a rest had lowest values. The least mean value of luminance is seen in taking a break, the last one being the darkest among the five tasks (see Table 2 and the left one in Figure 10).

Color temperature is a way to describe the light appearance provided by a light source. It gives us what the look and feel of the light produced are. It is measured in degrees of Kelvin (K). It ranges between 2000 and $6500 \mathrm{~K}$. At the lower end of the scale $(2000-3000 \mathrm{~K})$, the light produced is called warm white, and it provides an ambience of cozy, calm and intimate. The middle range of the scale $(3100-4500 \mathrm{~K})$ is referred to as cool white and its appearance is like bright and vibrant. A high value of color temperature above $4500 \mathrm{~K}$ is called daylight and it provides crisp invigorating ambience like daylight. Participants could have freedom to select a value between 2200 and $6500 \mathrm{~K}$ with Philip Hue. The mean values in the experiment varied between $3195 \mathrm{~K}$ and $5172 \mathrm{~K}$ depending upon the task. The influence of color temperature is slightly different than that of illuminance. That is, color temperature has the highest value in playing Sudoku among the five situations (i.e., cool-white). However, this is followed by reading a magazine and having dinner, whose values are similar in terms of color temperature (i.e., neutral-white). The mean value of color temperature is lowest when taking a break, and watching YouTube (i.e., warm-white) has a little higher mean value than taking a break (see Table 2 and the right one in Figure 10).

Table 2 Means and Standard Deviations of Illuminance and color temperature between tasks of lighting ( $\mathrm{n}=20)$

\begin{tabular}{lllll} 
Task & Illuminance (lux) & \multicolumn{2}{l}{ Color temperature (K) } \\
& M & SD & M & SD \\
\hline Playing Sudoku (T1) & 133.92 & 63.99 & 5172.95 & 1351.91 \\
\hline Reading a magazine (T2) & 46.36 & 14.38 & 4552.35 & 1387.03 \\
\hline Watching YouTube (T3) & 12.94 & 10.89 & 3534.75 & 2260.70 \\
\hline Having dinner (T4) & 72.30 & 33.41 & 4346.05 & 971.96 \\
\hline Taking a break (T5) & 4.47 & 3.96 & 3195.35 & 2202.42 \\
\hline
\end{tabular}
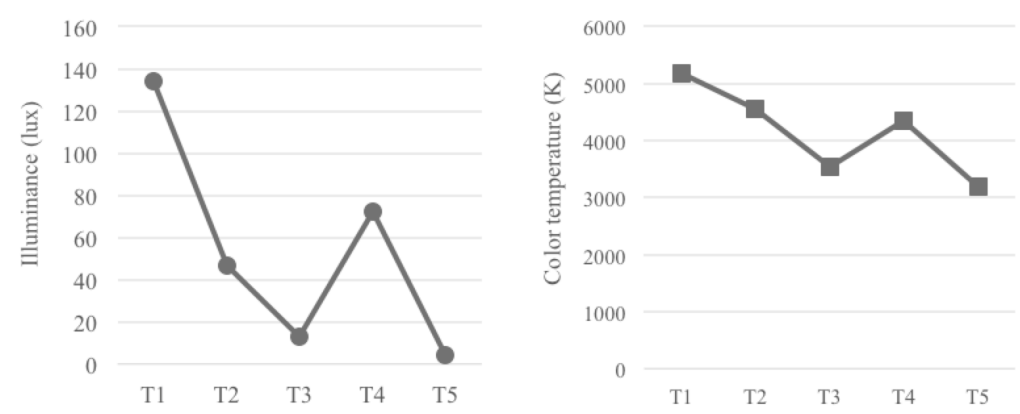

Figure 10 The mean value variation of illuminance (left) and color temperature (right) between situations 
Except for two tasks, watching Youtube and having dinner, the overall results indicate that tasks requiring a high level of concentration led to high illuminance or a high value in colour temperature. Low illuminance or low value in colour temperature were associated with tasks requiring much less concentration.

\section{2. The social effect of illuminance and color temperature}

A paired-sample t-test was conducted to evaluate the social effect on illuminance. There was a statistically significant increase in the illuminance of lighting for having dinner from single users $(\mathrm{M}=48.06, \mathrm{SD}=17.99)$ to couple users $[\mathrm{M}=96.53, \mathrm{SD}=26.96, \mathrm{t}(18)=-4.73, \mathrm{p}<.0005]$.

The differences seem significant only in having dinner between the single user group and the couple user group (see Table 3 and the left one in Figure 11). The couple user group tends to use higher illumination of lighting in having dinner than single user group. There was no significant difference between the two groups when they played Sudoku, read a magazine, watched YouTube and took a rest.

A paired-sample t-test was conducted to evaluate the social effect on colour temperature. There is no statistical significance between social effect and colour temperature. However, it seems that social effect might have influenced color temperature particularly in watching YouTube if the significant level considered $90 \%$ of the standard normal distribution of the sample: single users $(\mathrm{M}=4501, \mathrm{SD}=1806)$ and couple users $[\mathrm{M}=2568, \mathrm{SD}=2334, \mathrm{t}(18)=2.07$, $\mathrm{p}<.001]$.

According to the analysis, the single user group prefers higher values than the couple user group when they watched YouTube in terms of colour temperature (see Table4 and the right one in Figure 11). It means that the single user group uses more cool-white lighting while the couple user group prefers more warm-white.

Table 3 Illuminance differences between single and couple user groups

\begin{tabular}{lllllll} 
Task & \multicolumn{2}{l}{ Illuminance (lux) } & \multicolumn{2}{l}{ Color temperature (K) } & df & t \\
& M & SD & M & SD & & \\
& & & & & \\
Playing Sudoku (T1) & 149.42 & 65.82 & 118.42 & 61.46 & 18 & 1.09 \\
\hline Reading a magazine (T2) & 41.05 & 10.70 & 51.66 & 16.12 & 18 & -1.73 \\
\hline Watching YouTube (T3) & 16.17 & 10.94 & 9.70 & 10.36 & 18 & 1.36 \\
\hline Having dinner (T4) & 48.06 & 17.99 & 96.53 & 26.96 & 18 & $-4.73^{\star \star \star}$ \\
\hline Taking a break (T5) & 4.06 & 4.17 & 4.87 & 3.92 & 18 & -.45 \\
\hline
\end{tabular}

Table 4 Color temperature differences between single user and couple user groups

\begin{tabular}{lllllll} 
Task & \multicolumn{2}{l}{ Illuminance (lux) } & \multicolumn{2}{l}{ Color temperature (K) } & df & t \\
& M & SD & M & SD & & \\
Playing Sudoku (T1) & 5174.40 & 1302.59 & 5171.50 & 1470.26 & 18 & .01 \\
\hline Reading a magazine (T2) & 4338.20 & 1732.17 & 4766.50 & 979.35 & 18 & -.68 \\
\hline Watching YouTube (T3) & 4501.00 & 1807.00 & 2568.50 & 2334.40 & 18 & 2.07 \\
\hline Having dinner (T4) & 4614.40 & 826.42 & 4077.70 & 1073.03 & 18 & 1.25 \\
\hline Taking a break (T5) & 2764.30 & 2104.51 & 3626.40 & 233.44 & 18 & -.87
\end{tabular}



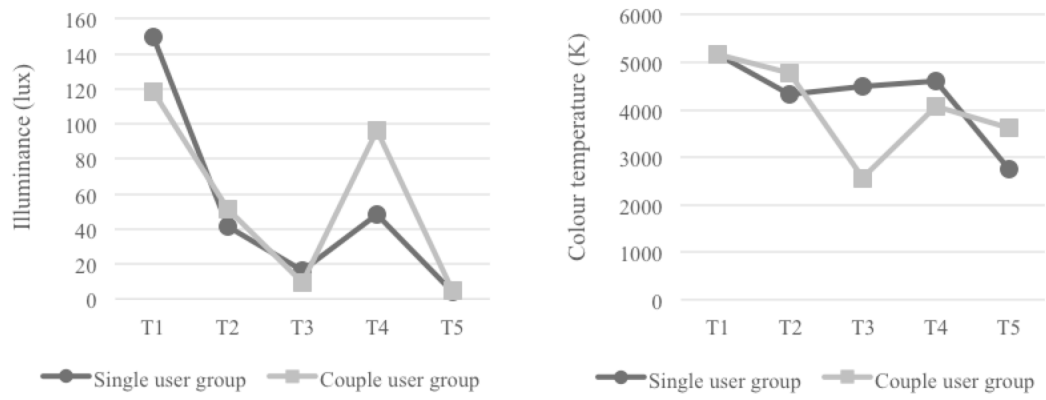

Figure 11 The mean value comparison of illuminance and color temperature between single and couple user groups

\section{3. The effect of level of concentration and social interaction on hue}

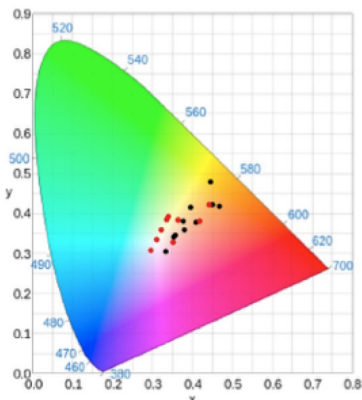

Task 1: playing Sudoku

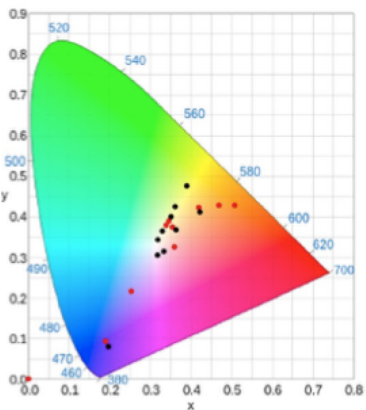

Task 3: watching YouTube

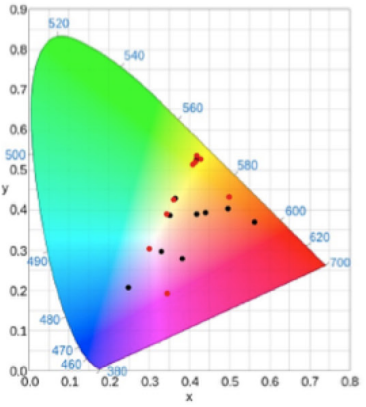

Task 5: taking a rest

Figure 12 hue distribution of five tasks on color coordinate

(black dots represent single user group and red dots represent couple user group)

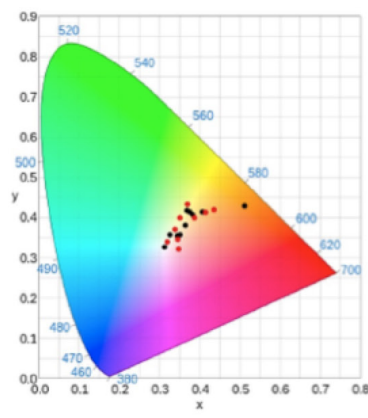

Task 2: reading a magazine

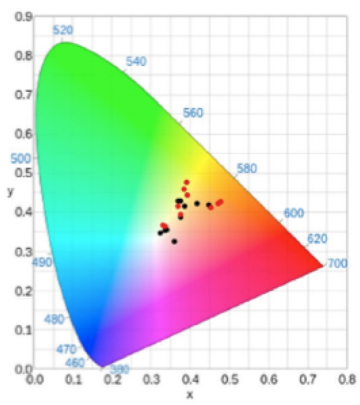

Task 4: having dinner 
Since hue, measured from the illuminometer, has its own coordinate values, hue is represented on the hue coordinate plane. The location of a hue coordinate represents the color: as it is closer to the top left area it goes more green, as it is closer to the bottom-left area it goes more blue, and as it is closer to the bottom-right it goes to more red. The hue coordinate of the lighting chosen in each situation is illustrated with points in Figure 12: black dots refer to the single user group, and red dots to the couple user group.

Comparing the distribution of dots between tasks, it turned out that the pattern is dispersedly distributed in both watching YouTube and taking a rest while the clustered pattern of distribution is characteristic for reading a magazine and having dinner. When participants read a magazine, they preferred yellow and red hues. A similar pattern is observed in the task of having dinner. In the case of playing Sudoku, the distribution of dots is a bit more scattered than reading a magazine and having dinner, but interestingly the hue ranges from green and yellow to red (see Figure 12).

Looking into the difference between single users and couple users on a task, the outstanding difference appeared in watching YouTube and taking a break. In watching YouTube, the single user group's hue is clustered between yellow and red, but the couple user group's hue is dynamically spread between blue and red. When the participants took a break, the hues of single users are spread between blue and red, while those of couple users are located between green and red. In playing Sudoku, the dots of a single user group are dispersed within red but those of the couple user group are mainly distributed between green and red hue. In both reading a magazine and having dinner, no significant difference was seen between single user group and couple user group (see Figure 12). However, no particular pattern of hue distribution was observed between tasks in terms of the level of concentration.

\section{4. The reasons why particular lightings were chosen per task}

According to the results, people adjusted Philips Hue to provide the optimal lighting conditions to perform the tasks rather than out of personal preference. However, the reasons vary between tasks (Figure 13). For instance, 'good to concentrate' is most often mentioned as the reason for choosing a particular lighting when they play Sudoku. 'Increasing visibility' gets top priority as a reason why they adjust the light in case of reading a magazine. When they watch YouTube, light is adjusted mainly out of consideration for how much 'comfort to eyes' is given, and to how good for concentration it is. As they have dinner, about half of the participants set particular lighting parameters because they thought lighting provides 'mood' to help stimulate their appetite. 'Increasing visibility' of food is also an important reason for their lighting selection. In the case of taking a rest, providing 'mood for relaxing themselves' is the most important reason for setting up the lighting atmosphere. However, a few participants said that they set up lighting just because they are used to that setting without particular reasons (i.e., familiarity). This was reported in the tasks such as playing Sudoku, watching YouTube, and having dinner, but not in the situation of reading a magazine and taking a rest.

There are some differences between the single and couple user groups in terms of reasons for choosing a particular light setting (see also Figure 13). For instance, concentration is the only reason raised by the couple user group in the situation of reading a magazine. In the situation of watching YouTube, concentration is the most important reason for their setting up the 
lighting to the couple user group, while visual comfort is the main reason for the single user group. A similar pattern was also observed in the situation of having dinner: visibility is most often mentioned, which is followed by mood for the couple user group while the opposite is true for single users. In addition to that, consideration for the other was third-ranked and reported only by the couple user group. In the situation of taking a rest, the mood is the only reason for the couple users' lighting selection. However, for the single user group, visual comfort and concentration are also important reasons.

Task 1: Playing Sudoku

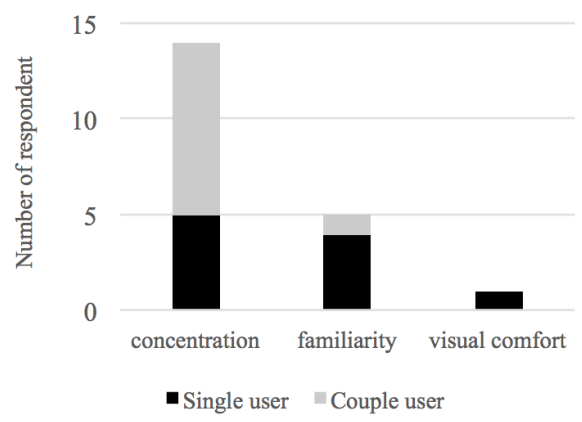

Task 3: Watching YouTube

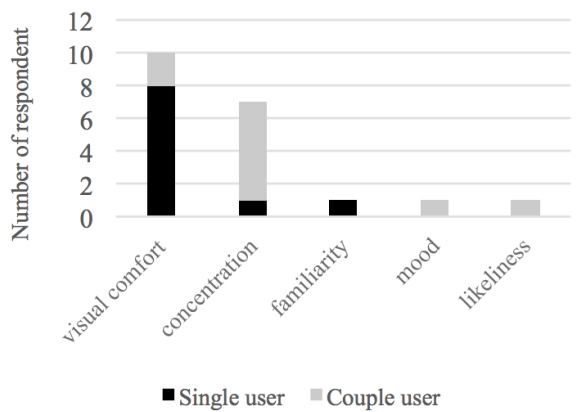

Task 5: Taking a rest

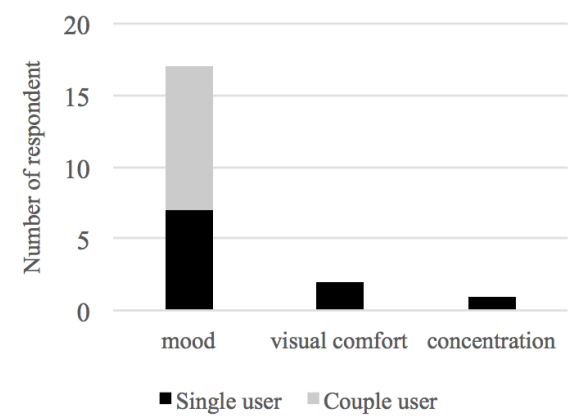

Task 2: Reading a magazine

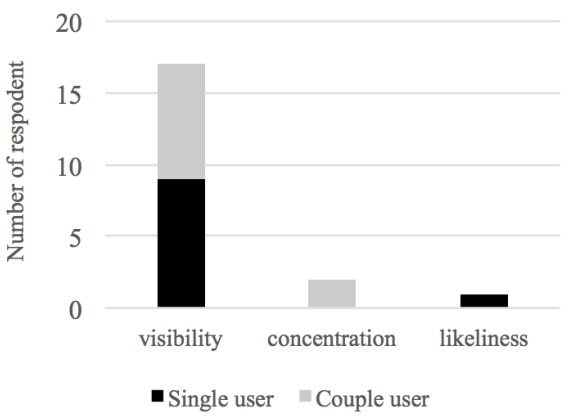

Task 4: Having dinner

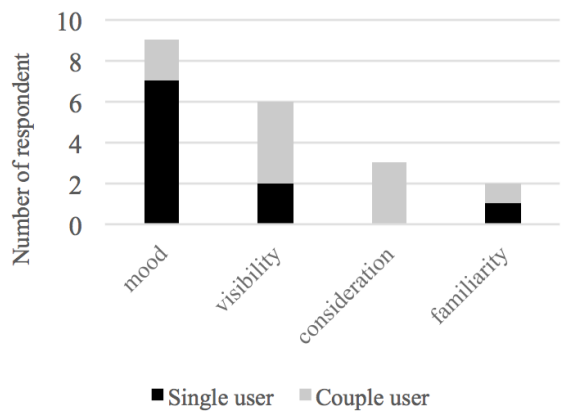

Figure 13 The differences of reasons of setting up particular lighting parameters between tasks 


\section{Discussion and Conclusions}

This study attempted to reveal how the level of concentration required in a particular task and social factor influence the manipulation of lighting parameters in home lighting experience, particularly focusing on the major ones of lighting such as illuminance, colour temperature and hue. With the findings of the study, it could contribute to provide a better understanding of at-home positive lighting experience in terms of the relationship between the level of concentration, social effect and lighting parameters with design practitioners. If these aspects are considered in the lighting product development process, it would lead to more satisfactory user experience for lighting products and services such as Philips Hue, interactive lighting system, smart lighting system. For instance, it has not taken the characteristics of an at-home situation in terms of concentration and social effect into consideration in the use of Philips Hue. If a smart lighting system could be aware of the situation where the user is, the system could provide her/him/them with ideal lighting experience best for the situation by automatically tuning lighting parameters.

The overall findings of the study indicate that illuminance, colour temperature, and hue are significantly dependent upon the level of concentration required in at-home lighting use, and also have only a partial dependence on social effect. This implies that although we assumed that people have their own personal preference of lighting, their preference can be to a large extent dependent on the degree of concentration required in at-home pleasant lighting use. Hence, there are common patterns between people in manipulating lighting parameters, which are less dependent on personal differences.

Since it has not been much investigated in which way lighting parameters are related to the level of concentration in at-home pleasant lighting use, the five tasks with different situations were chosen for the experiment based on the extent to which they are focused. The results indicate that at-home lighting use requiring a lot of concentration leads to high illuminance or high value in colour temperature. In the same vein, low illuminance or low value in colour temperature can be associated with situations requiring much less concentration. However, no particular pattern of hue distribution was observed between tasks given in the study. Interestingly, it is peculiar that the participants still preferred high illuminance and high value in colour temperature only in the situation of having dinner, which requires relatively low concentration. Assumably, this is because people tend to avoid any influence on visual perception made by lighting when they eat meals as one of the essential activities for human survival although it does not require a high degree of concentration. Also in the interview, mood and visibility were mentioned by participants as possible influential factors. This could support the assumption as well. In case of the task watching youtube, an iPad was used, and the device already offers necessary amount of light. Probably, this could give an explanation of why low illuminance and relatively low value of color temperature were preferred by the participants although the task itself is meant to require high concentration. Although the focus in the study was on the concentration aspect, other factors also seem to play a role as the results of the interviews show: the experimental setting was not meant to investigate which factors influence the lighting parameters. Only in the interviews after the experiment participants were asked for those factors.

Our assumption about social effects of lighting use was that whether being alone or together 
with somebody else could make differences in setting lighting parameters because lighting could also function as an emotional trigger in a space. In other words, we could imagine that couple users would pay more attention to making an atmosphere where they are than a single user group. However, the result that social factors does not seem to have a great effect on manipulating lighting parameters is striking: in illuminance, it seems effective only in the case of having dinner; in colour temperature, only with watching YouTube; and in hue, its influence is seen only in watching YouTube and taking a rest. A possible explanation for why social effects are not significant in all the tasks given in the study, is that humans probably have common patterns of lighting use depending on particular situations, which work more strongly than whether they are alone or with someone else. Another explanation is that the experiment was conducted in a laboratory which is not exactly the same as the participants' homes, which might influence their behaviour.

Apart from the influence of level of concentration and social effect in at-home pleasant lighting use, it is also interesting to figure out the reasons behind the selection of particular lightings made by people. It turned out that people actually determine particular lighting based on the primary goal that they are supposed to achieve in a particular situation although the reasons vary between tasks. This seems to override personal preference of lighting. This can also explain why lighting use is more dependent on the level of concentration required in at-home pleasant lighting use than on the preference of a user himself/herself. According to the result, the influence of social factor on lighting use is not strong, regardless of how much concentration a task requires.

Offermans (2016) proposed a three-layer framework for designing the interactions for intelligent lighting, called Layered Light Interaction Model. The three layers are lamps, lighting, and context. Based on the model, those parameters could be divided into lamp layer (intensity, color, texture, shape) and lighting layer (area, movement, distribution). Since only the intensity and color were selected for investigation in this study, the scope seems limited only on the lamp level. Therefore, it is necessary to take the lighting level into account in further studies.

Although the study led to some interesting findings on the influence of level of concentration and social effect in lighting parameters, the sample size was not large enough to gain more reliable and strong outcomes. In addition to that, the results might be specific for only this age group and cultural background, considering that a generation experiences similar lifestyles. For instance, the hierarchical nature of Korean culture might have influenced the decision making behaviour of those in the couples' experiment. Therefore, a follow-up study should take more participants, preferably more generations and more diverse cultures into account. Furthermore, a next study should investigate the factors involved in choosing lighting in the home, both functional aspects such as visibility and concentration, as well as aspects of mood such as valence and arousal.

\section{References}

1. Blühm, A. and Lippincott, L. (2001). Light! The Industrial Age 1750-1900. Art \& Science, Technology \& Society. Thames \& Hudson, New York.

2. Chang, M. (2010). Role and usage of light in product interface design. (Maste's thesis), Kookmin University.

3. Choi, S., Kim, H., Kang, S., \& Nam, S. (2007). Interior Architectural Lighting. South Korea: Kimundaing. 
4. Desmet, P. M. A., Vastenburg, M. H., Van Bel, D., \& Romero Herrera, N. (2012). Pick-A-Mood: Development and application of a pictorial mood-reporting instrument. In J. Brassett, P. Hekkert, G. Ludden, M. Malpass, \& J. McDonnell (Eds.). Proceedings of the 8th International Conference on Design and Emotion [CD ROM]. London, UK: Central Saint Martin College of Art \& Design.

5. Elizabeth, T. (1996). MIT Research - Brain Processing of Visual Information. Retrieved December 19 , 1996, from http://bit.ly/smlcHO.

6. Essig, L. (2005). Lighting and the Design Idea (2nd ed.), Wadsworth, Inc.

7. Essig, L., \& Setlow, J. (2012). Lighting and the design idea (3rd ed.): Wadsworth Cengage Learning.

8. Gilette, J. (2012). Theatrical Design and Production: An Introduction to Scene Design and Construction, Lighting, Sound, Costume, and Makeup (7th ed.): McGrawHill Connect Learn Succeed.

9. Goldstein, E. (2009). Sensation and perception: Cengage Learning.

10. Hoonhout, J., Jumpertz, L., \& Mason, J. (2011). The future of interaction with light and lighting dynamics. Interaction Techniques for Future Lighting Systems, 14.

11. Jun, D., \& Kim, C. (2014). The application of lighting factors in information transfer: a case study of external hard disk. Paper presented at the 2014 Fall Conference of the Ergonomics Society of Korea, South Korea.

12. Jun, D., Kim, C., \& Kwak, Y. (2014). Exploring the Role of Lighting in Human-Product Interaction. Paper presented at the The 1st Asian Conference on Ergonomics and Design, Jeju, South Korea.

13. Knoop, M. (2006). Dynamic lighting for well-being in work places: Addressing the visual, emotional and biological aspects of lighting design. In Proceedings of the 15th international symposium lighting engineering. Lighting Engineering Society of Slovenia, Bled, Slovenia (pp. 6374).

14. Magielse, R., \& Ross, P. R. (2011, September). A design approach to socially adaptive lighting environments. In Proceedings of the 9th ACM SIGCHI Italian Chapter International Conference on Computer-Human Interaction: Facing Complexity (pp. 171-176). ACM.

15. McCaffety, K. (2006). The chandelier through the centuries. Savoy House.

16. Offermans, S. A. M. (2016). Interacting with light (Doctoral dissertations). Eindhoven University of Technology, Eindhoven, the Netherlands.

17. Offermans, S. A. M., van Essen, H. A., \& Eggen, J. H. (2014). User interaction with everyday lighting systems. Personal and Ubiquitous Computing, 18(8), 2035-2055. http://doi.org/10.1007 /s00779-014-0759-2.

18. Sanders, M. S., \& McCormick, E. J. (1993). Human factors in engineering and design (7th ed.): McGRAW-HILL book company.

19. Watson, D., \& Clarck, L. A. (1994). The panas-x: Manual for the positive and negative affect schedule - expanded form. Iowa, IA: The University of lowa.

20. Watson, D., Clark, L. A., \& Tellegen, A. (1988). Development and validation of brief measures of positive and negative affect: The panas scales. Journal of Personality and Social Psychology, 54(6), 1063-1070. 